\title{
Generation of electron high energy beams with a ring-like structure by a dual stage laser wakefield accelerator
}

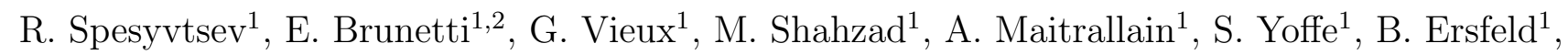
A. Kornaszewski ${ }^{1}$, M.J.V. Streeter ${ }^{3,4,5}$, O. Finlay ${ }^{3}$, Y. Ma ${ }^{3,4,6}$, B. Kettle ${ }^{5}$, S.J.D. Dann ${ }^{3,4}$, F. Albert $^{7}$, N. Bourgeois ${ }^{8}$, S. Cipiccia ${ }^{9}$, J.M. Cole ${ }^{5}$, E. Gerstmayr ${ }^{5}$, I.G. González ${ }^{10}$, A. Higginbotham $^{11}$, A.E. Hussein 6 , K. Falk ${ }^{12}$, K. Krushelnick 6 , N. Lemos ${ }^{7}$, N.C. Lopes ${ }^{5,13}$, C.

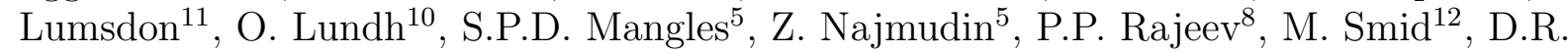
Symes $^{8}$, A.G.R. Thomas ${ }^{3,4,6}$, and D.A. Jaroszynski ${ }^{1,2}$

${ }^{1}$ SUPA, Department of Physics, University of Strathclyde, Glasgow G4 0NG, UK

${ }^{2}$ The Cockcroft Institute, Keckwick Lane, Daresbury, WA4 4AD, United Kingdom

${ }^{3}$ Physics Department, Lancaster University, Lancaster LA1 4YB, United Kingdom

${ }^{4}$ The Cockcroft Institute, Keckwick Lane, Daresbury, WA4 4AD, United Kingdom

${ }^{5}$ The John Adams Institute for Accelerator Science, Imperial College London, London, SW7 $2 \mathrm{AZ}, \mathrm{UK}$

${ }^{6}$ Center for Ultrafast Optical Science, University of Michigan, Ann Arbor, MI 48109-2099, USA

${ }^{7}$ Lawrence Livermore National Laboratory, Livermore, CA 94550, USA

${ }^{8}$ Central Laser Facility, STFC Rutherford Appleton Laboratory, Didcot OX11 0QX, UK

${ }^{9}$ Diamond Light Source, Harwell Science and Innovation Campus, Fermi Avenue, Didcot OX11 ODE, UK

${ }^{10}$ Department of Physics, Lund University, P.O. Box 118, S-22100, Lund, Sweden

${ }^{11}$ York Plasma Institute, Department of Physics, University of York, York YO10 5DD, UK

${ }^{12}$ ELI Beamline, Institute of Physics of the ASCR, Na Slovance 2, Prague 182 21, Czech Republic

${ }^{13}$ GoLP/Instituto de Plasmas e Fuso Nuclear, Instituto Superior Tcnico, U.L., Lisboa 1049-001, Portugal

\begin{abstract}
The laser wake-field accelerator (LWFA) traditionally produces high brightness, quasi-monoenergetic electron beams with Gaussian-like spatial and angular distributions. In the present work we investigate the generation of ultra-relativistic beams with ring-like structures in the blowout regime of the LWFA using a dual stage accelerator. A density down-ramp triggers injection after the first stage and is used to produce ring-like electron spectra in the $300-600 \mathrm{MeV}$ energy range. These well defined, annular beams are observed simultaneously with the on-axis, high energy electron beams, with a divergence of a few milliradians. The rings have quasi-monoenergetic energy spectra with an RMS spread estimated to be less than 5\%. Particle-in-cell simulations confirm that off-axis injection provides the electrons with the initial transverse momentum necessary to undertake distinct betatron oscillations within the plasma bubble during their acceleration process.
\end{abstract}

Keywords: Laser wake-field accelerator, electron accelerator, plasma physics, high energy electrons

Further author information: (Send correspondence to D.A. Jaroszynski)

D.A.J.: E-mail: d.a.jaroszynski@strath.ac.uk

R.S.: E-mail: roman.spesyvtsev@strath.ac.uk 


\section{INTRODUCTION}

Since the first demonstration of controlled acceleration of monoenergetic high energy electron beams by the laser wake-field accelerator (LWFA), ${ }^{1-3}$ their use has become widespread. They now form the basis of table-top sources of both high brightness, high energy ultrashort pulsed electron beams ${ }^{4-6}$ and secondary radiation..$^{7-9}$ An ultra-short, relativistically intense laser pulse focused into a low-density plasma can create a plasma wake that accelerates electrons to high energies within a few millimeters of propagation distance. ${ }^{10,11}$ Moreover, a simple combined injector and accelerator scheme can be implemented in the LWFA blowout regime, where the ponderomotive force of the intense driving laser pulse produces a fully evacuated plasma bubble while creating the conditions for electron self-injection. ${ }^{12}$ Self-injection can be controlled by either the driving laser pulse, ${ }^{13-15}$ or by shaping the plasma density profile. ${ }^{6,13,16}$ A common way to control self-injection is using a density downramp. ${ }^{16,17}$ In this case the plasma bubble structure expands - providing a stable condition for self-injection. Electrons are then injected into the back of the bubble and accelerate rapidly.

The LWFA usually produces one or more on-axis beams with low divergence. ${ }^{4,18}$ Here, we show experimentally that a stable electron ring structure can be formed at injection and subsequently accelerated to about $0.5 \mathrm{GeV}$ with excellent shot-to-shot repeatability and about $5 \%$ energy spread. The ring structure is correlated with strong X-ray emission because of the large amplitude betatron oscillations. ${ }^{19}$ This dependence provides a promising means of optimising betatron radiation for applications. PIC simulations confirm that off-axis injection and large amplitude oscillations are associated with the electron rings. Their previous observations have been attributed to two different mechanisms..$^{20,21}$ In the first study they were attributed to electrons trapped in toroidal pockets located outside the bubble, ${ }^{20}$ while in the second study the rings were attributed to injection into the bubble itself. ${ }^{21}$ Electrons are briefly injected into the bubble along the plasma density down-ramp, forming a well-localised annular distribution of high energy electrons as they undergo betatron oscillations. ${ }^{21}$ The injection mechanism substantially increases the X-ray flux ${ }^{19,20}$ because the number of photons emitted by an electron is proportional to the transverse momentum ${ }^{9}$ and the critical photon energy is proportional to the cube of the transverse momentum. ${ }^{9}$ In addition, it can provide a method of producing primary and secondary radiation with unusual spatial and temporal characteristics. For example, the phenomena allows to directly generate ring-like X-ray beams without the need for any optical components.

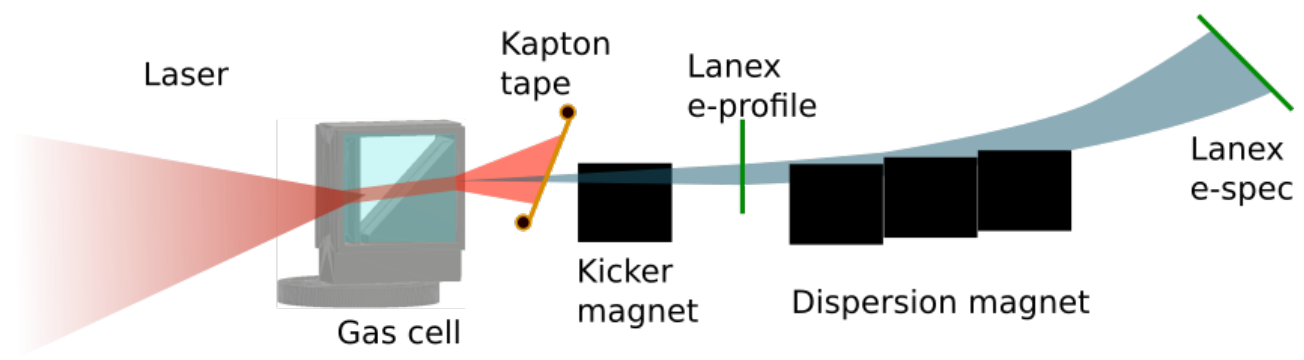

Figure 1. Experimental setup layout. High power laser pulse is focused with a f/40 off-axis parabolic mirror into a gas cell. The gas cell has two stages designed to provide high plasma density at the laser entrance, for electron injection, and a low plasma density flat region for acceleration to high energies. The electrons were self-injected using the down-ramp injection mechanism between the two cells. ${ }^{20}$ The laser is blocked after the gas cell by a gold coated kapton tape. Electron energy spectra are measured using a magnet dispersion section, a LANEX screen and a CCD camera. In addition, the electron beam profile is characterised before the dispersion section using a LANEX screen and a CCD camera.

\section{LWFA EXPERIMENT}

\subsection{EXPERIMENTAL SETUP}

The experiment has been carried out using the ASTRA-GEMINI laser at the Central Laser Facility, Rutherford Appleton Laboratory. The layout of the experimental setup showing the main components is presented in Figure 1. 
Laser. High power ultra-short laser pulses are derived from a Ti:sapphire based laser system. Laser energy after the amplifier is measured on every shot, which was on average $12.3 \pm 0.3 \mathrm{~J}$. The total efficiency of the beam transport to the target area has been measured to be $51 \%$, thus $6.3 \mathrm{~J}$ is delivered to the target area. The laser beam is focused with a f/40 off-axis parabolic mirror to a beam size of $46 \pm 1 \mu \mathrm{m}$ (FWHM), corresponding to a normalised vector potential $a_{0}=1.3$. The laser spectrum measured after compression is centred at a wavelength of $797 \pm 0.8 \mathrm{~nm}$ and has a FWHM width of $27.6 \pm 1.4 \mathrm{~nm}$. The laser pulse duration of $40 \pm 5 \mathrm{fs}$, measured using frequency-resolved optical gating (FROG) setup, is close to the bandwidth limited pulse duration of 34 fs.

Gas target. A two-stage gas cell is used for injection and acceleration of electrons. The gas target cell is 3D printed, comprising two cells, where the first cell length is $3 \mathrm{~mm}$ while the second cell length can be varied from 2 to $20 \mathrm{~mm}$. The cell is mounted on a XYZ-translation stage, which enables optimisation of the gas cell length. The first stage is filled with a mixture of $\mathrm{He}+\mathrm{N}_{2}(2 \%)$ for electron injection, while the second stage is filled with pure He for electron acceleration. The cells are separated by a $1 \mathrm{~mm}$ thick wall with a centrally positioned vertical slit of $0.5 \mathrm{~mm}$. The end wall of the second cell is angled at 45 degrees to the front wall. This geometry allows adjustment of the length of the flat gas density region, corresponding to the acceleration stage, which is achieved by changing the height of the gas cell with respect to the laser propagation axis. The pressure in both gas cells can be varied independently. The plasma density is determined from spectra of Raman side scattered laser radiation. The plasma density, $n_{e}$, is found to depend linearly on the backing pressure, $P, n_{e}\left(10^{18} \mathrm{~cm}^{-3}\right)=4.1 \times P($ bar $)$, which was determined from offline calibration measurements performed using a stretched laser pulse to avoid any dependence on the normalised vector potential $a_{0} .{ }^{22}$ A typical backing gas pressure of 480 mbar (giving a plasma density of $2 \times 10^{18} \mathrm{~cm}^{-3}$ ) was used during the electron spectra measurements presented in section 2.3.

Beamline and diagnostics. The residual transmitted laser beam is blocked by a gold coated kapton tape located after the gas cell. It is mounted on a tape drive that translates after every shot to ensure a fresh area of the tape blocks the laser beam. A kicker magnet separates the electron and X-ray beams for X-ray phase contrast imaging studies. ${ }^{19}$ The kicker magnet is positioned in the beamline for the electron profile measurements presented in section 2.2. The electron beam profile is monitored by a LANEX scintillator screen placed before the main dispersive section and imaged on a CCD camera. The electron beam energy is measured in a spectrometer comprising a $1 \mathrm{~T}$ dipole magnet that deflects the beam onto a LANEX scintillator screen imaged by a CCD camera. The setup geometry and measured magnetic field map allows the electron energy spectrum to be reconstructed using a particle tracking method.

\subsection{ELECTRON PROFILE MEASUREMENTS}

The electron beam profile is measured after the kicker magnet (with the magnet located in the beam path). Two main features are clearly observed during the measurements: a central beam and a ring as shown in Figure 2. The ring structures sporadically appear when the pressure in the second cell exceeds 700 mbar and up to a pressure of 1000 mbar $\left(2.7-4.1 \times 10^{18} \mathrm{~cm}^{-3}\right)$. These observed structures are independent of the pressure in the first cell. One reason for this may be that injection is triggered by the down-ramp density region created at the entrance of the second cell due to gas flow dynamics due to the pressure difference between the two cells. Electron rings are observed for second stage lengths of $12-18 \mathrm{~mm}$. The divergence of the rings varies between 30 and $60 \mathrm{mrad}$, and the thickness of a single ring is about $3 \mathrm{mrad}$. However, in some cases more broad features are observed, including multiple rings with displaced centres.

\subsection{ELECTRON SPECTRUM MEASUREMENTS}

Electron energy spectra are measured after the dispersive section when the kicker magnet and the electron profile monitor are removed from the beam path. A single run with 23 consecutive shots is displayed in Figure 3, where electron spectra are measured for a plasma density of $2 \times 10^{18} \mathrm{~cm}^{-3}$ and a $17 \mathrm{~mm}$ acceleration length.

A typical spectrum, as illustrated in Figure 4, shows a broad energy spectrum with a low divergence of about $1.8 \mathrm{mrad}$ at FWHM superimposed on a ring shape profile that has a conical full-angle of about $14.5 \mathrm{mrad}$. The broad energy spectrum peaks at about $1.0 \pm 0.14 \mathrm{GeV}$ while the ring structure occurs at about $500 \mathrm{MeV}$. The energy spread of the rings must be very small because the structure is preserved through the spectrometer. The ring FWHM thickness in $x$ direction is $4.3 \mathrm{mrad}$ and in $y$ direction is $15 \mathrm{mrad}$. Since the $y$ axis is in the dispersion direction we anticipate an increase in the ring thickness due to the electron energy spread of the conical beam. 

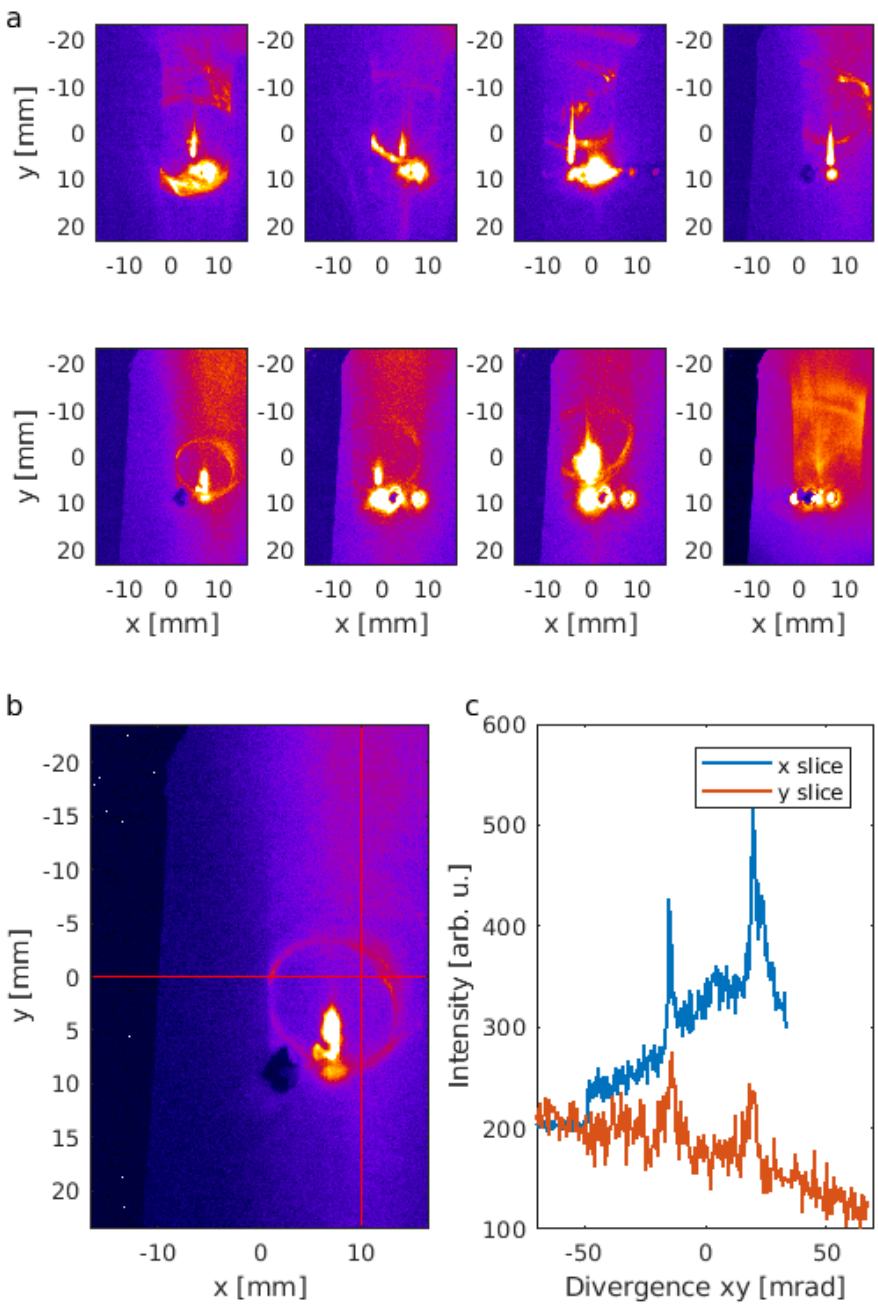

Figure 2. Electron beam profile measured on the LANEX e-profile monitor. (a) shows selected shots over a 2-day period with examples of electron ring images. (b) shows an one example with cross-sections (c) taken along the indicated lines. The ring opening full-angle in (b) is $35 \mathrm{mrad}$ and the ring FWHM thickness is about $3 \mathrm{mrad}$. The full opening angle of the ring fluctuated from shot to shot between 30 and $60 \mathrm{mrad}$.

\section{PIC SIMULATIONS}

In order to better understand the physics behind the ring formation we have carried out Particle-In-Cell (PIC) simulations using the FBPIC (FourierBessel Particle-In-Cell) code. ${ }^{23}$ Input gas density profile for the simulations is obtained from gas flow calculations using the ANSYS Fluent software with the gas cell design geometry. The absolute gas densities are then linearly scaled to match the experimental parameters. The laser beam waist is set to $36 \mu \mathrm{m} \mathrm{1/} e^{2}$ radius, the FWHM pulse duration to $40 \mathrm{fs}$ and a pure He gas is used in both cells. Several simulations are performed for a range of input parameters until a clear ring formation is observed in the electron distribution. Figure 5 shows a result of the PIC simulations after $10 \mathrm{~mm}$ propagation distance in the gas cell. The overall energy spectrum (Figure 5 (a)) is broad and peaks at around $1.2 \mathrm{GeV}$ in agreement with the experimental results. In addition, a clear annular structure is observed in the electron angular distribution, as can be seen in 


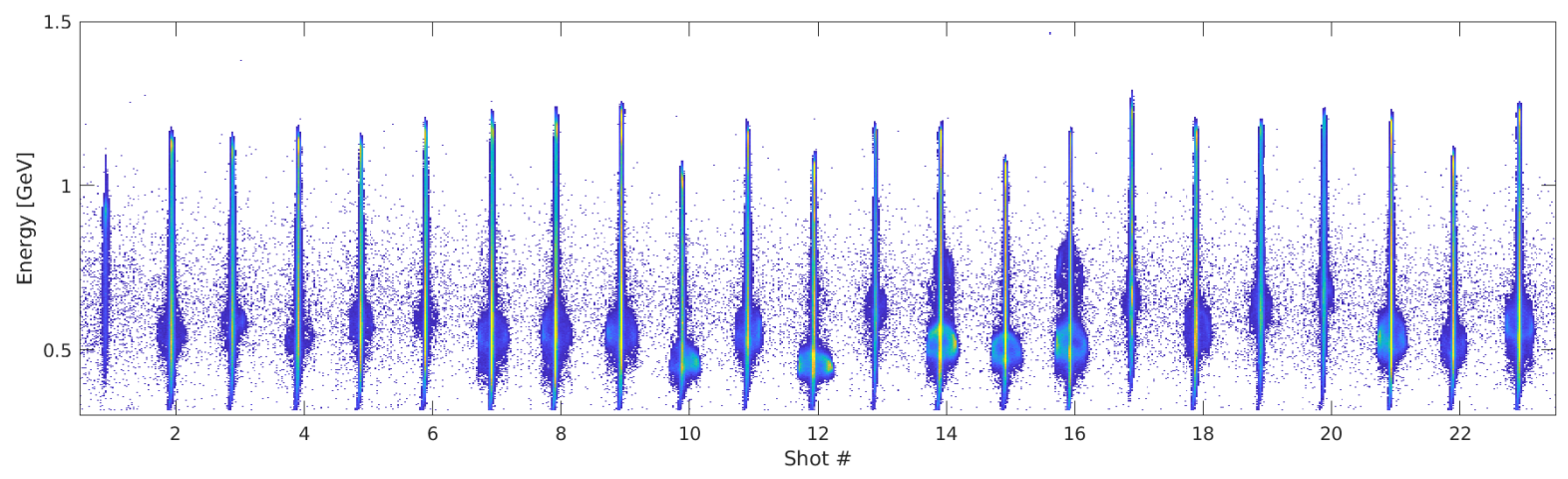

Figure 3. Electron energy spectrum measurements after the dispersion section. 23 consecutive shots are displayed. The broad energy distribution is observed simultaneously with the quasi-monoenergetic ring structure, which occurs on almost every shot.

Figure 5 (b). These electrons have a narrow energy spread and are localised within the plasma bubble as shown in Figure 5 (d-f). The bubble diameter for these simulation parameters (plasma density of $1.75 \times 10^{18} \mathrm{~cm}^{-3}$ and $\left.\mathrm{a}_{0}=1\right)$ is about $20 \mu \mathrm{m}$.

Electrons within the ring distributions are briefly injected off-axis with a considerable initial transverse momentum, and rapidly accelerate to about $200 \mathrm{MeV}$. During the acceleration electrons undergo betatron oscillations with an amplitude of about $5 \mu \mathrm{m}$, but remain within the plasma bubble. This process continues until they outrun the bubble due to the velocity mismatch and start to decelerate. These electrons are localised in transverse phase-space and upon far field propagation will form a spatially circular structure because they all exit with the same transverse momentum, which is experimentally observed on the beam profile monitor.

\section{DISCUSSION}

FBPIC simulations are in a good qualitative agreement with the experimental observations. The annular electron distribution has a narrow energy spread that agrees with the fact that dipole magnet does not distort the ring shape. The broad energy spectrum of the on-axis accelerated electrons indicates continuous electron injection
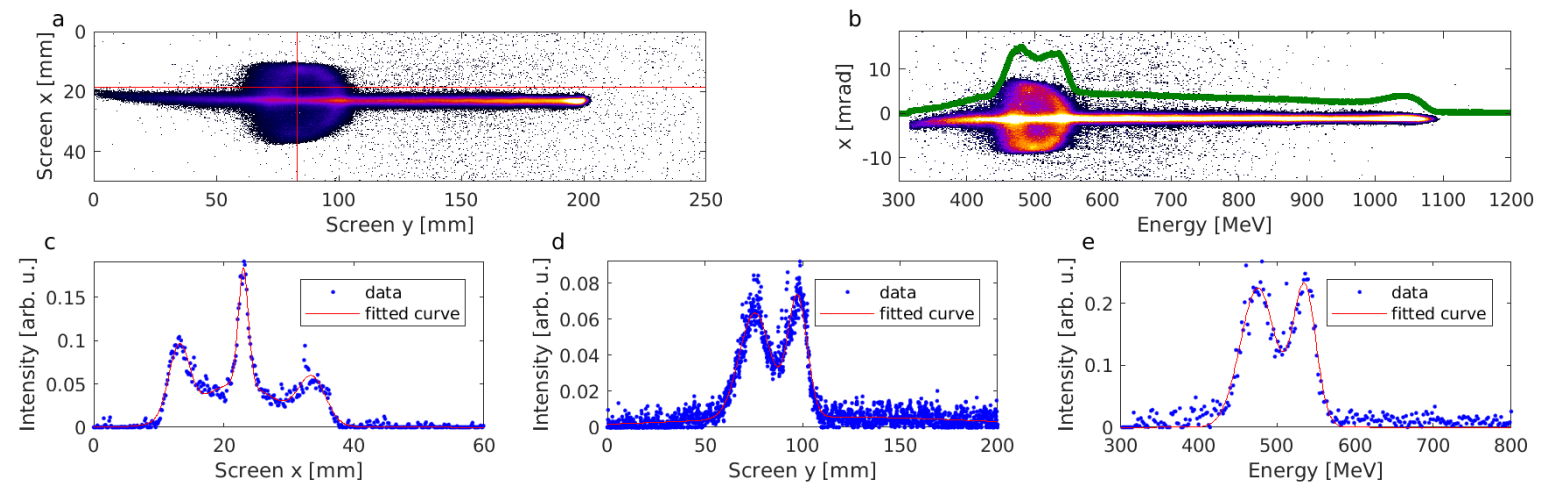

Figure 4. (a) A typical shot captured on the electron spectrometer LANEX screen and (b) corresponding calibrated energy spectrum and angle showing a circular electron distribution centred around $500 \mathrm{MeV}$. The green curve outlines the electron density integrated over the $x$ (non-dispersive) coordinate. The cross-section along the red lines depicted in (a) are presented in (c) along $x$ direction and in (d) along $y$ direction (the dispersion axis). (e) The same data as in (d) but using a calibrated energy axis. The profiles are fitted with multiple Gaussians to extract the divergence and energy spread for different sections of the beam. The on-axis beam FWHM divergence is 1.8 mrad, the ring FWHM thickness in $x$ (non-dispersive) direction is $4.3 \mathrm{mrad}$ and in $y$ (dispersive) direction is $15 \mathrm{mrad}$. 

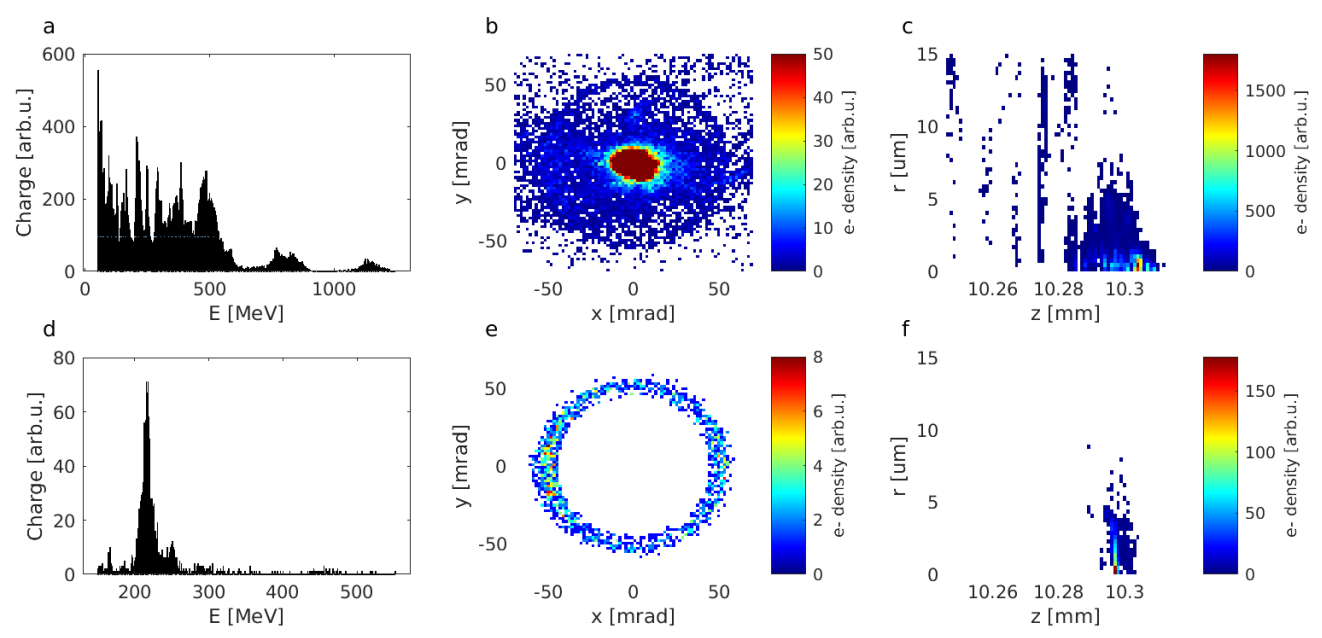

Figure 5. Electron beam parameters after $10 \mathrm{~mm}$ propagation in the gas cell. Complete electron energy spectrum (a), divergence (b) and position (c) distributions. Laser propagates along the $\mathrm{z}$ axis. Electron energy spectrum (d), divergence (e) and position (f) distribution for electrons within a divergence cone between 45 and $60 \mathrm{mrad}$.

and acceleration inside the plasma bubble. On the other hand, the annular electron structure has a quasimonoenergetic energy distribution, which corresponds to a singular localised injection event. The simulations confirm the formation of such an electron bunch with a narrow energy spread. The divergence of the annular structure is comparable with previously observed annular features. ${ }^{20,21}$ While the ring conical opening angle is much wider than the divergence of the on-axis beam, the ring thickness is comparable to the on-axis beam divergence. Thus the actual transverse phase-space volume of the ring is similar to the phase space distribution of the on-axis beam. Since the energy spread of the annular beam is also low, it is therefore a good quality beam that should be suitable for a long range transport.

Electron rings generation is also correlated with an increased flux in betatron X-ray emission, which has been discussed in ref $\left[{ }^{19}\right]$, and is in agreement with the results reported earlier. ${ }^{21}$ The plasma bubble produces both longitudinal acceleration fields and transverse focusing fields. This provides not only rapid acceleration but also large amplitude betatron oscillations of the injected electrons due to off-axis injection, which explains the increase in the X-ray flux when rings are observed. The peak energy of the on-axis electron beam always exceeds that of the ring. The energy of the annular beam is lower in simulations compared with the experimental results, which is most likely due to a mismatch in the gas density profile used in the simulations. Further adjustments of the input parameters for FBPIC simulations should resolve this discrepancy and also would provide a valuable feedback for the future optimisation of experimental parameters.

\section{ACKNOWLEDGMENTS}

We acknowledge support of the UK STFC core grants ST/P002056/1 (Cockcroft Institute), ST/P000835/1 (John AdamsInstitute), and the United States Department of Energy Grant No. DE-NA0002372. This work was partially supported by EuPRAXIA (Grant No. 653782), ECs LASERLAB-EUROPE (Grant No. 654148), U.K. EPSRC Grant No. EP/J018171/1,EP/J500094/1 and EP/N028694/1), EuCARD-2 (Grant No. 312453), the Extreme Light Infrastructure (ELI) European Project, and by FCT - Fundacao para a Ciencia e a Tecnologia,15/17 Ministerio da Ciencia e Ensino Superior, Portugal under the contract POCI/FIS/59574/2004 and by the auspices of the USDepartment of Energy by Lawrence Livermore National Laboratory under the contract DE-AC52- 07NA27344, as well asLawrence Livermore National Security, LLC, and DOE Early Career Research Prog. SCW1575/1. LLNL- JRNL-742178.

Data associated with this paper is available at: https://doi.org/10.15129/7394020e-191b-4362-965e-dbe54adb521d 


\section{REFERENCES}

[1] Mangles, S. P. D., Murphy, C. D., Najmudin, Z., Thomas, A. G. R., Collier, J. L., Dangor, A. E., Divall, E. J., Foster, P. S., Gallacher, J. G., Hooker, C. J., Jaroszynski, D. A., Langley, A. J., Mori, W. B., Norreys, P. A., Tsung, F. S., Viskup, R., Walton, B. R., and Krushelnick, K., "Monoenergetic beams of relativistic electrons from intense laser-plasma interactions," Nature 431, 535 (Sep 2004).

[2] Geddes, C. G. R., Toth, C., van Tilborg, J., Esarey, E., Schroeder, C. B., Bruhwiler, D., Nieter, C., Cary, J., and Leemans, W. P., "High-quality electron beams from a laser wakefield accelerator using plasma-channel guiding," Nature 431, 538 (Sep 2004).

[3] Faure, J., Glinec, Y., Pukhov, A., Kiselev, S., Gordienko, S., Lefebvre, E., Rousseau, J.-P., Burgy, F., and Malka, V., "A laser-plasma accelerator producing monoenergetic electron beams," Nature 431, 541 (Sep 2004).

[4] Brunetti, E., Shanks, R. P., Manahan, G. G., Islam, M. R., Ersfeld, B., Anania, M. P., Cipiccia, S., Issac, R. C., Raj, G., Vieux, G., Welsh, G. H., Wiggins, S. M., and Jaroszynski, D. A., "Low emittance, high brilliance relativistic electron beams from a laser-plasma accelerator," Phys. Rev. Lett. 105, 215007 (Nov 2010).

[5] Leemans, W. P., Gonsalves, A. J., Mao, H.-S., Nakamura, K., Benedetti, C., Schroeder, C. B., Tóth, C., Daniels, J., Mittelberger, D. E., Bulanov, S. S., Vay, J.-L., Geddes, C. G. R., and Esarey, E., "Multi-gev electron beams from capillary-discharge-guided subpetawatt laser pulses in the self-trapping regime," Phys. Rev. Lett. 113, 245002 (Dec 2014).

[6] He, Z.-H., Hou, B., Nees, J. A., Easter, J. H., Faure, J., Krushelnick, K., and Thomas, A. G. R., "High repetition-rate wakefield electron source generated by few-millijoule, 30 fs laser pulses on a density downramp," New J. Phys 15, 053016 (May 2013).

[7] Corde, S., Ta Phuoc, K., Lambert, G., Fitour, R., Malka, V., Rousse, A., Beck, A., and Lefebvre, E., "Femtosecond x rays from laser-plasma accelerators," Rev. Mod. Phys. 85, 1-48 (Jan 2013).

[8] Cipiccia, S., Wiggins, S. M., Shanks, R. P., Islam, M. R., Vieux, G., Issac, R. C., Brunetti, E., Ersfeld, B., Welsh, G. H., Anania, M. P., Maneuski, D., Lemos, N. R. C., Bendoyro, R. A., Rajeev, P. P., Foster, P., Bourgeois, N., Ibbotson, T. P. A., Walker, P. A., Shea, V. O., Dias, J. M., and Jaroszynski, D. A., "A tuneable ultra-compact high-power, ultra-short pulsed, bright gamma-ray source based on bremsstrahlung radiation from laser-plasma accelerated electrons," J. Appl. Phys. 111(6), 063302 (2012).

[9] Cipiccia, S., Islam, M. R., Ersfeld, B., Shanks, R. P., Brunetti, E., Vieux, G., Yang, X., Issac, R. C., Wiggins, S. M., Welsh, G. H., Anania, M.-P., Maneuski, D., Montgomery, R., Smith, G., Hoek, M., Hamilton, D. J., Lemos, N. R. C., Symes, D., Rajeev, P. P., Shea, V. O., Dias, J. M., and Jaroszynski, D. A., "Gamma-rays from harmonically resonant betatron oscillations in a plasma wake," Nat. Phys 7, 867 (Sep 2011).

[10] Malka, V., "Laser plasma accelerators," Phys. Plasmas 19(5), 055501 (2012).

[11] Esarey, E., Schroeder, C. B., and Leemans, W. P., "Physics of laser-driven plasma-based electron accelerators," Rev. Mod. Phys. 81, 1229-1285 (Aug 2009).

[12] Kalmykov, S. Y., Beck, A., Yi, S. A., Khudik, V. N., Downer, M. C., Lefebvre, E., Shadwick, B. A., and Umstadter, D. P., "Electron self-injection into an evolving plasma bubble: Quasi-monoenergetic laser-plasma acceleration in the blowout regime," Phys. Plasmas 18(5), 056704 (2011).

[13] Gonsalves, A. J., Nakamura, K., Lin, C., Panasenko, D., Shiraishi, S., Sokollik, T., Benedetti, C., Schroeder, C. B., Geddes, C. G. R., van Tilborg, J., Osterhoff, J., Esarey, E., Toth, C., and Leemans, W. P., "Tunable laser plasma accelerator based on longitudinal density tailoring," Nat. Phys 7, 862 (Aug 2011).

[14] Thomas, A. G. R., Najmudin, Z., Mangles, S. P. D., Murphy, C. D., Dangor, A. E., Kamperidis, C., Lancaster, K. L., Mori, W. B., Norreys, P. A., Rozmus, W., and Krushelnick, K., "Effect of laser-focusing conditions on propagation and monoenergetic electron production in laser-wakefield accelerators," Phys. Rev. Lett. 98, 095004 (Mar 2007).

[15] Islam, M. R., Brunetti, E., Shanks, R. P., Ersfeld, B., Issac, R. C., Cipiccia, S., Anania, M. P., Welsh, G. H., Wiggins, S. M., Noble, A., Cairns, R. A., Raj, G., and Jaroszynski, D. A., "Near-threshold electron injection in the laser-plasma wakefield accelerator leading to femtosecond bunches," New J. Phys 17, 093033 (Sep 2015). 
[16] Tooley, M. P., Ersfeld, B., Yoffe, S. R., Noble, A., Brunetti, E., Sheng, Z. M., Islam, M. R., and Jaroszynski, D. A., "Towards attosecond high-energy electron bunches: Controlling self-injection in laser-wakefield accelerators through plasma-density modulation," Phys. Rev. Lett. 119, 044801 (Jul 2017).

[17] Swanson, K. K., Tsai, H.-E., Barber, S. K., Lehe, R., Mao, H.-S., Steinke, S., van Tilborg, J., Nakamura, K., Geddes, C. G. R., Schroeder, C. B., Esarey, E., and Leemans, W. P., "Control of tunable, monoenergetic laser-plasma-accelerated electron beams using a shock-induced density downramp injector," Phys. Rev. Accel. Beams 20, 051301 (May 2017).

[18] Manahan, G. G., Brunetti, E., Shanks, R. P., Islam, M. R., Ersfeld, B., Anania, M. P., Cipiccia, S., Issac, R. C., Raj, G., Vieux, G., Welsh, G. H., Wiggins, S. M., and Jaroszynski, D. A., "High resolution, single shot emittance measurement of relativistic electrons from laser-driven accelerator," in [Proc. SPIE 8079, Laser Acceleration of Electrons, Protons, and Ions; and Medical Applications of Laser-Generated Secondary Sources of Radiation and Particles], 8079 (2011).

[19] Hussein, A. E., Senabulya, N., Ma, Y., Streeter, M. J. V., Kettle, B., Dann, S. J. D., Albert, F., Bourgeois, N., Cipiccia, S., Cole, J. M., Finlay, O., Gerstmayr, E., González, I. G., Higginbotham, A., Jaroszynski, D. A., Falk, K., Krushelnick, K., Lemos, N., Lopes, N. C., Lumsdon, C., Lundh, O., Mangles, S. P. D., Najmudin, Z., Rajeev, P. P., Schlepütz, C. M., Shahzad, M., Smid, M., Spesyvtsev, R., Symes, D. R., Vieux, G., Willingale, L., Wood, J. C., Shahani, A. J., and Thomas, A. G. R., "Laser-wakefield accelerators for high-resolution x-ray imaging of complex microstructures," Sci. Rep 9(1), 3249 (2019).

[20] Pollock, B. B., Tsung, F. S., Albert, F., Shaw, J. L., Clayton, C. E., Davidson, A., Lemos, N., Marsh, K. A., Pak, A., Ralph, J. E., Mori, W. B., and Joshi, C., "Formation of ultrarelativistic electron rings from a laser-wakefield accelerator," Phys. Rev. Lett. 115, 055004 (Jul 2015).

[21] Zhao, T. Z., Behm, K., Dong, C. F., Davoine, X., Kalmykov, S. Y., Petrov, V., Chvykov, V., Cummings, P., Hou, B., Maksimchuk, A., Nees, J. A., Yanovsky, V., Thomas, A. G. R., and Krushelnick, K., "Highflux femtosecond x-ray emission from controlled generation of annular electron beams in a laser wakefield accelerator," Phys. Rev. Lett. 117, 094801 (Aug 2016).

[22] Matsuoka, T., McGuffey, C., Cummings, P. G., Horovitz, Y., Dollar, F., Chvykov, V., Kalintchenko, G., Rousseau, P., Yanovsky, V., Bulanov, S. S., Thomas, A. G. R., Maksimchuk, A., and Krushelnick, K., "Stimulated raman side scattering in laser wakefield acceleration," Phys. Rev. Lett. 105, 034801 (Jul 2010).

[23] Lehe, R., Kirchen, M., Andriyash, I. A., Godfrey, B. B., and Vay, J.-L., "A spectral, quasi-cylindrical and dispersion-free particle-in-cell algorithm," Comput. Phys. Commun 203, 66 - 82 (2016). 